attributable to the non-specialist format requested from contributors and to the inclusion of extra papers to fill gaps in the conference material.

The papers fall into four sections, starting with "Solar Observations and Theories", moving out through the "Interplanetary Medium" so consideration of "Geophysical Responses to Solar Activity" and completed by studies of "Progress in Forecasting Technology". J. W. Evans leads off with his "Introductory Review of Solar Activity", a highly competent and concise account of solar phenomena and nomenclature for the newcomer to the field, spoilt only by some lack of attention to numerical detail and descriptive clarity in places. Further papers in section 1 divide equally between observa. tions of large and small scale solar magnetic field distributions and of flare particle, radio, ultraviolet and $\mathrm{X}$-rayline emissions, rounded off by Sturrock's résumé of magnetic flare models. Chief omissions are adequate discussion of hard X-ray observations (an important flare diagnostic) and explicit description of optical flare characteristics other than inference of flare fields from $\mathrm{H} \alpha$ filtergrams.

Continuing through the papers on interplanetary and geophysical aspects, the reader encounters a continuing high quality and density of information. Particularly valuable to the newcomer are the reviews of the solar wind by Dryer and Cuperman and of magnetospheric particles by Paulikas. Despite all this material, however, a shadow of the stamp-collecting era remains looming over the science of solar activity. Application of games theory (Smith) and of Markov chains (Mogilevsky) gives "successful" activity predictions for only a few days, little better than suitably "accurate" and "skilful" subjective classification of active regions. (Predictions of heliographic locations of activity are more satisfactory.)

Thus, barring a spectacular improvement in these techniques or a conceptual breakthrough in activity theories, McIntosh and Dryer's editorial anticipation of the military, astronautic and ecological benefits to accrue from activity predictions must be, at best, long-term ideals. Viewed, however, as a unifying account of solar activity, their book can be recommended to all.

JohN C. Brown

\section{Critique in Palaeontology}

Models in Paleobiology. By Thomas J. M. Schopf. Pp. vi +250 . (Freeman, Cooper: San Francisco, 1972.) \$9.50.

AT last we have been provided with a thought-provoking text on invertebrate palaeontology. Tom Schopf is to be congratulated not only on having the vision and temerity to question the motivation and direction of palaeontological enquiry but also for producing this timely panoramic critique at a comparatively moderate price.

The volume comprises ten essays on various aspects of palaeontological analysis and synthesis grouped under the headings of (1) "Models in Paleobiology", (2) "Morphology", (3) "Population and Evolution", and (4) "Distribution". They result from a 1971 symposium on models in palaeobiology jointly sponsored by the Paleontological Society and Geological Society of America at their annual general meeting in Washington DC. The styles of the presentations vary from author to author but each is introduced by a pertinent précis by Schopf. Thus no matter how complex and abstruse some of the articles may at first seem, Schopf has succeeded in ensuring that all contributions are intelligible even to those geologists who suffer from minimal and/or inappropriate scientific backgrounds. This is no mean feat since the discussions liberally impinge on the cognate sciences of evolutionary palaeontography, biogeochemistry and statistics.

Schopf's implied secondary theme that all too frequently the last decade has witnessed an explosion in unquestioning and uncanalized palaeontography is hard to refute. In assembling these papers he has effected a heroic job which ought to revitalize the science and its utility. Even now there are deficiencies which are a salutary reflexion on the inadequacies and inappropriateness of our scientific trainings. Too often we are trained to see the trees at the expense of seeing the wood. The advances quoted in this volume are frequently simple and derive from using the methodology of a cognate science rather than a large body of complementary subject matter. The basic deficiency underlying all the themes is our bland neglect of the physiological parameters which have a profound influence on both biogeochemistry and ecology, which thereby affect evolutionary palaeontography and culminate in the difficulties which arise in statistical analysis as a result of cumulative omission. This does not mean that statistics should be ignored as unsound, but that it should be treated with the reverent constructive caution so admirably displayed by Simberlof. For, as Eldredge and Gould so rightly, but damningly, imply, we tend to report those things for which we have explanations while ignoring those aspects which embarrass us. This is perhaps epitomized in Dodd and Schopf's truism that the biogeochemical data available to us are woefully inadequate as the geochemical results have seldom tested the intimate relationships which would have been required had the enquiries rested on sound biological principles. This can easily be seen to result from the fact that all too frequently undergraduate geologists align themselves with either the physical sciences or the biological sciences but seldom both. Thus, quite apart from the intrinsic value of the variety of approaches clearly analysed, the volume makes salutary reading for those concerned with both educational and applied aspects of geology and the earth sciences.

This book should be compulsory reading for all geologists and earth scientists who direct departments and assess research proposals. It will provide palaeontologists with many happy hours of brain-teasing, stimulation and self-assessment. It should intrigue the curious undergraduate, absorb the graduate student, and infuse panic in the hearts of those palaeontographers who have got stuck in a rut. No geology department library can afford to be without this volume, for its intellectual applications are not limited to palaeontology alone. This tome has achieved for palaeontology an analogy of what the Geophysical Laboratory, Washington DC, contributed to petrology some decades ago.

\section{J. A. E. B. Hubbard}

\section{Vibrations in Fluid}

Sound, Structures and Their Interaction. By Miguel C. Junger and David Feit. Pp. $x+470$. (The MIT: Cambridge, Massachusetts and London, October 1972.) $\$ 27.50$.

THERE are many books on acoustics and many on vibrations. Some even consider the radiation of sound from vibrating surfaces and others the vibration induced in a structure by an imping. ing sound wave. There is, however, no book devoted to the interaction between structures and the surrounding fluid. At best a chapter or two in existing texts might be devoted to particular aspects of the interaction. The majority of written work on this topic is spread through the technical literature, and not a small portion of this is classified because of its underwater naval applications. Dr Junger himself has been responsible for several significant developments in underwater acoustics.

This textbook is the first book to draw together much of this material and to present it in a coherent way. The book arose from the need, at postgraduate level, of a text on the interaction between a vibrating structure and the surrounding medium. The interaction becomes most important with dense fluids such as water, but there are cases in which even the weaker coupling with air is important. The results will also be important for containment structures such as gas cooled 